\title{
Spatiotemporal Heterogeneity Across Metastases and Organ- Specific Response Informs Drug Efficacy and Patient Survival in Colorectal Cancer
}

\author{
Jiawei Zhou ${ }^{1}$, Quefeng $\mathrm{Li}^{2}$, Yanguang $\mathrm{CaO}^{1,3}$ \\ ${ }^{1}$ Division of Pharmacotherapy and Experimental Therapeutics, School of Pharmacy, University of \\ North Carolina at Chapel Hill, NC 27599, USA; \\ ${ }^{2}$ School of Public Health, University of North Carolina at Chapel Hill, NC 27599, USA; \\ ${ }^{3}$ Lineberger Comprehensive Cancer Center, School of Medicine, University of North Carolina at \\ Chapel Hill, Chapel Hill, NC 27599, USA
}

\begin{abstract}
The sum of target lesions is routinely used to evaluate patient objective responses to treatment in the RECIST criteria, but it fails to address response heterogeneity across metastases. This study argues that spatiotemporal heterogeneity across metastases and organ-specific response is informative for drug efficacy and patient survival. We analyzed the longitudinal data of 11,404 metastatic lesions in 2,802 colorectal cancer patients from five Phase III clinical trials. Initially, a metric Gower distance was applied to quantify response heterogeneity across metastases. Next, the spatiotemporal response heterogeneity across anatomical sites, therapies, and $K R A S$ mutation status were assessed and examined for their association with drug efficacy and long-term patient survival. The response of metastatic lesions broadly differed across anatomical sites and therapies. About $60 \%$ of patients had at least one lesion respond contrarily from total tumor size. High interlesion heterogeneity was associated with shorter progression-free survival and overall survival. Targeted therapies (bevacizumab or panitumumab) combined with standard chemotherapy reduced inter-lesion heterogeneity and elicited more favorable effects from liver lesions $(\mathrm{p}<0.001)$ than chemotherapy alone. Moreover, the favorable responses in liver metastases (> 30\% shrinkage) were associated with extended patient overall survival ( $\mathrm{p}<0.001$ ), in contrast to lesions in the lungs and lymph nodes. Altogether, the spatiotemporal response heterogeneity across metastases informed drug efficacy and patient survival, which could improve the current methods for treatment evaluation and patient prognosis.
\end{abstract}

\section{Keywords}

metastases; response heterogeneity; colorectal cancer; patient survival; RECIST

Corresponding author: Yanguang Cao, Ph.D., Division of Pharmacotherapy and Experimental Therapeutics, UNC School of Pharmacy, 2318 Kerr Hall, UNC Eshelman School of Pharmacy, Chapel Hill, NC 27599-7569, yanguang@ unc.edu, Phone: +1-919-966-4040.

Conflicts of interest: The authors declare that there is no conflict of interest regarding the publication of this article 


\section{Introduction}

The systemic spread of cancer cells from primary site to distant organs is the leading cause of cancer modality, accounting for about $90 \%$ of cancer deaths (1). Preventing metastasis remains the main but challenging objective in cancer therapy (2). Although originating from the same primary tumor, metastatic lesions at different anatomical sites usually possess different tumor characteristics $(3,4)$, due to divergent somatic mutations, epigenetic alterations, and organ-specific microenvironments $(5,6)$. The genomic and phylogenetic heterogeneity across metastatic lesions have been illustrated in-depth using genomic sequencing data coupled with advanced computational approaches $(7,8)$. However, the phenotypic heterogeneity in response to interventions across metastases and its correlation with patient prognosis has not been well characterized, which is the focus in our study.

The magnitude of therapeutic efficacy is routinely quantified based on overall tumor burden, calculated by the sum of all target lesions according to the Response Evaluation Criteria in Solid Tumors (RECIST) v1.1 (9). The RECIST guidelines, adopted in most clinical trials, do not account for the response disparities across metastatic lesions and may not be optimal for targeted therapies (10). Unlike conventional cytotoxic therapy, the responses of targeted therapies and acquired resistance are often lesion-specific (11). In a study of non-small cell lung cancers, the benefits of nivolumab varied broadly across metastatic sites. The efficacy was more pronounced in the lymph nodes (LN) lesions than in the liver and bone lesions (12). In another study, the patterns of response and progression displayed high lesionspecific variation in $B R A F$-mutant metastatic melanoma patients treated with a combination of two MAPK targeted therapies, dabrafenib and trametinib (13). Response variations across anatomical sites could result from divergent clonal genetic, epigenetic, and transcriptional features (14), conferred by different evolutionary pressures and organ-specific tumor microenvironments (5). Using the sum of target lesions may conceal the unique phenotypes of each lesion and the lesion-selective effect of targeted therapy, thus fail to disclose insights into lesion-specific response and resistance. We argue that the quantitative assessment of the spatiotemporal response across metastatic lesions can add another dimension to tumor heterogeneity and resistance, thereby deepening the investigation of the organ-specific factors that shape the phenotypes of metastases.

Most literature studies investigated the inter-metastasis heterogeneity through the distinct genotypic and phenotypic profiles, including genetic mutations, RNA expression, cell proliferation, and metastatic potential. $(6,12,13,15-18)$ Our study, for the first time, characterized the full response dynamics of each lesion and corroborated their response heterogeneity to inform long-term patient survival. In our analysis, we considered colorectal cancer (CRC) as the tumor system. CRC has a high prevalence and mortality and is expected to cause approximately 53,200 deaths in 2020 (19). The five-year survival rate is only $13.1 \%$ for metastatic colorectal cancer (mCRC) in contrast to $90.1 \%$ for localized CRC patients (20). Many advances have been made in CRC treatment, with the use of targeted therapies combined with standard chemotherapies. Targeted therapies, including anti-EGFR and antiVEGF antibodies and recently approved anti-PD-1/PD-L1 immunotherapies, provided a durable response and prolonged patient survival in a selected group of patients (21). This study incorporated longitudinal data from five Phase III trials, comprising 2,802 mCRC 
patients with 11,404 metastatic lesions. We examined the spatiotemporal response heterogeneity across metastases in either chemotherapy alone or combined with targeted therapies. We corroborated the associations between lesion-specific response and patient progression-free survival (PFS), overall survival (OS), and $K R A S$ status. Our findings supported the substantial significance of including spatiotemporal response dynamics across metastases for the improved evaluation of drug efficacy and patient prognosis.

\section{Materials and Methods}

\section{Data sources}

The data was extracted from the Project Data Sphere using the following criteria: stage III or IV mCRC patients; individual lesion longitudinally measured; and recorded patient OS. In total, five Phase III trials (registration number: NCT00339183, NCT00115765, NCT00364013, NCT00272051, NCT00305188) encompassing 2,802 patients with 11,404 metastatic lesions were selected. Only 28 patients in the FOLFOX arm did not have PFS information and were excluded from the PFS analysis. The clinical trial information is summarized in Supplementary Table 1. The study protocols were approved by institutional review boards at each participating center and conducted with the principles of the Declaration of Helsinki. All patients provided written informed consent before study-related procedures were performed. We obtained the full access of data from Project Data Sphere database.

\section{Patient demographics and clinical characteristics}

Patient demographic information including age, gender, race, body surface area (BSA), lactate dehydrogenase (LDH), ECOG performance score, $K R A S$ status, primary tumor type, metastatic organs and number, prior medical history, RECIST response, and treatment types are listed in Table 1. The RECIST criteria-defined target lesions were also included in the analysis. Lesion anatomical sites with total lesion numbers below 50 were pooled into an "other" group including adrenal glands, chest, kidney, pancreas, peritoneum, skin, spleen, genitourinary/reproductive, and others (not specified).

\section{Lesion response dynamics}

We developed an algorithm in MATLAB R2017b to characterize the response dynamics and the duration of the response, stable, and relapse phases at a lesion-specific level (Supplementary Figure 1, Supplementary Codes). The criteria for defining the response, stable, and relapse phases were selected according to the RECIST v1.1. Specifically, the duration from the tumor baseline until the tumor size decreased 30\% was defined as the response phase. The period, from which the lesion regrew more than $120 \%$ until the last sampling point, was described as the relapse phase. The period of neither response nor relapse was defined as the stable phase. We did not consider the mixed responses in the derivation of Gower distance, as only very few lesions showed mixed response in our dataset. 


\section{Gower distance}

The lesion-specific response patterns were described by five variables: three binary variables representing the presence of the response, stable, or relapse phase, and two continuous variables indicating the tumor nadir ratio and last ratio (tumor nadir size and last size before the end of trial normalized by tumor baseline). The Gower distance (22) of these five variables was computed as a dissimilarity measure of the response patterns across the metastases within each patient. For comparing the response patterns for lesion $i$ and lesion $j$, the formula of Gower distance is

$$
G_{i j}=\frac{\sum_{v} \delta_{i j v} \cdot d_{i j v}}{\sum_{v} \delta_{i j v}}
$$

Where $v$ is a variable describing the lesion response patterns, including the response, stable, relapse phase as well as the tumor nadir and last ratio $\delta_{i j v}$. is a binary indicator equal to one when both lesions are not missing for variable $v$, otherwise equal to zero. As all the lesions in our dataset were not missing any variables, $\sum_{V} \delta_{i j v}$ is the number of variables and equal to five.

$$
\begin{aligned}
& \text { For binary variables } v, d_{i j v}=\left\{\begin{array}{c}
0, \text { if } x_{i v}=x_{j v} \\
1, \text { otherwise }
\end{array}\right\} \\
& \text { For continuous variables } v, d_{i j v}=\frac{\left|x_{i v}-x_{j v}\right|}{\left\{\max _{k}\left(x_{k v}\right)-\min _{k}\left(x_{k v}\right)\right\}}
\end{aligned}
$$

Where the equation $\max _{k}\left(x_{k V}\right)-\min _{k}\left(x_{k V}\right)$ represents the range of the variable $v$. The $d_{i j v}$ is set to zero if $\max _{k}\left(x_{k V}\right)-\min _{k}\left(x_{k V}\right)=0$.

Lesion-level heterogeneity was quantified by the Gower distances across the metastases. The average Gower distances of all the metastases within one patient represents the patient-level heterogeneity. The Gower distance does not require minimum numbers of metastatic lesions in the calculation. The Gower distance reveals the lesion-average degree of heterogeneity, and a high number of metastases do not necessarily result in a high estimate of Gower distance.

\section{Statistical analysis}

Comparisons of two continuous variables were performed using the Mann-Whitney test. For multiple comparisons, we performed the Kruskal-Wallis test, followed by Dunn's tests with Bonferroni corrections. PFS (defined as the start of therapies until RECIST-defined progression or death) and OS (defined as the start of therapies until patient death) among the groups were depicted using Kaplan-Meier curves and compared using log-rank tests. The estimated hazard ratio (HR) and $\mathrm{p}$ value of +Gower distance - controlling for other confounding factors - were calculated in the Cox proportional hazard model stratified by treatment type. The mean (standard deviation) and median (interquartile range) of each 
group compared are presented in Supplementary Tables 2-12, and a p value $<0.05$ with a two-sided alternative was considered statistically significant. All the statistical tests were performed using R 3.5.1 or GraphPad Prism 8. The figures were made in R 3.5.1, GraphPad Prism 8 and BioRender software.

\section{Results}

\section{The response dynamics were heterogeneous across metastatic lesions}

We evaluated the response dynamics of all individual metastatic lesions $(n=11,404)$, as well as the total tumor burden (the sum of the diameter of the target lesions within each patient) from 2,802 patients for comparison (Supplementary Figure 2). Patient demographics and clinical characteristics are summarized in Table 1 . The median number of metastases was 4 (range 1-20). The responses of the primary lesions were not included in our dataset since most patients had already received colectomy surgery before enrollment. We defined three responsive phases (response, stable, relapse) according to the RECIST v1.1 and developed an algorithm to quantify the durations of these phases (see Methods and Supplementary Figure 1). The responsive phases, together with the tumor nadir size (best response) and the last tumor size before the end of the trial (relapse rate), were used to assess the lesion-level response to therapy (Figure 1A). As shown in Figure 1B, the dynamics of the total tumor burden masked the individual lesion responses. For instance, in patient 508, the total tumor burden exhibited an initial response, then a stable response, and finally, the relapse phase, while the LN metastatic lesion remained in the stable phase throughout the treatment. In patient 501, the total tumor burden remained stable throughout the trial, while the abdomen lesion quickly relapsed, and the gastrointestinal (GI) tract lesion responded durably during the treatment. The response dynamics could also be different for lesions within the same organ, as shown in patient 1312. The patients whose lesions responded differently from the total tumor burden are summarized in Figure 1C. More than $60 \%$ of the patients $(1,764$ patients over 2,802 ) had at least one lesion responding differently from the total tumor burden. In addition, the fraction of lesions with differently responding dynamics varied across anatomical sites, with the lowest fraction in the liver and other organs (35\%) and the highest in the bone (48\%) (Figure 1D). The small fraction of differently responding lesions in the liver was mainly due to the large size and number of hepatic metastases dictating the trend of the total tumor burden. Collectively, these observations suggested that the response status of the total tumor burden could not fully reflect the response dynamics of individual lesions, as it failed to account for the lesion-specific response and resistance features.

\section{The heterogeneous responses across metastatic lesions predicted patient survival}

To quantify response heterogeneity, we computed the Gower distance (22), an index reflecting the degree of divergence in the response dynamics across metastases. Five parameters extracted from the lesion response profiles were used to calculate the lesion-level Gower distance, including three phases (response, stable, and relapse), tumor nadir ratio, and last ratio (see Methods and Figure 2A). Patient-level Gower distance was derived by averaging the lesion-level Gower distances for all metastases within each patient, and it was independent of the lesion number for patients with more than two lesions (tau $=0.005$ and $p$ $=0.71$ in Kendall correlation test). The lesion response dynamics of the patients with high or 
low Gower distance are shown in Supplementary Figure 3A and 3B, where a higher Gower distance implies a higher inter-lesion heterogeneity (ILH) in the response dynamics.

To examine the association between patient survival and ILH in the response dynamics, we stratified patients by their OS. The patients with higher OS had lower ILH in their response dynamics compared to those with relatively lower OS ( $p<0.0001$, Figure 2B). Similarly, the patients with high ILH ( $275^{\text {th }}$ quantile) had significantly worse OS (median 422 days) than those with medium ILH $\left(25^{\text {th }}-75^{\text {th }}\right.$ quantile, median OS 550 days $)$ or low ILH ( $\leq 25^{\text {th }}$ quantile, median OS 630 days) ( $p<0.0001$, Figure 2C). This observation was confirmed in the Cox proportional hazard model stratified by therapy (Gower distance HR=1.14, $\mathrm{p}<$ 0.001 in OS; HR=1.19, $\mathrm{p}<0.001$ in PFS), controlling the influence of the tumor baseline and the number of metastases (Figure 2D). The power of the Gower distance in survival prediction was further validated by performing 500 times of Cox regression on the synthesized datasets via bootstrap sampling (Figure 2E). Overall, the increased ILH was associated with worse survival outcomes for patients with mCRC. These findings aligned with previous studies showing that tumors with higher response heterogeneity were associated with worse survival $(23,24)$. We also evaluated the Gower distance's predictability to the post-progression survival (PPS). The significant difference of PPS in the two groups (low vs. high Gower distance) confirmed the Gower distance's predictability to patient long-term survival (Figure 2F).

Next, we explored the spatial heterogeneity by comparing the lesion-level Gower distances across metastatic organs. The organ with the highest Gower distance was the GI tract (median 0.28), and those with the lowest were the liver and the lungs (median 0.23) (Figure $2 \mathrm{G}$ and Supplementary Table 2). The ILH also differed within the treatment types. Targeted therapies, including bevacizumab or panitumumab, with the standard chemotherapies, showed marked reductions in ILH ( $\mathrm{p}<0.05$ ) compared to chemotherapy alone (Figure $2 \mathrm{H}$ and Supplementary Table 3).

\section{Targeted therapies reduced lesion response heterogeneity and exhibited favorable efficacy on hepatic metastases}

To further explore the response heterogeneity across treatments, we compared the ILH between the targeted therapies and chemotherapy alone. We analyzed the bevacizumab and the panitumumab arms to examine the lesion-level benefits conferred by the targeted therapies, as well as the resulting ILH. We analyzed 377 patients in the bevacizumab group, compared to the chemotherapy only group (769 patients in FOLFOX and 419 patients in FOLFIRI). As expected, the OS and PFS were significantly improved by bevacizumab ( $\mathrm{p}<$ 0.0001), which was the major evidence supporting bevacizumab's regulatory approval (Figure 3A). Patients with relatively lower Gower distance had significantly longer survival in the bevacizumab arm, reinforcing that a higher ILH is associated with worse survival (Figure 3B). Next, we tested whether the survival benefit conferred by bevacizumab was associated with a reduction in lesion response heterogeneity. Interestingly, despite notably lower lesion nadir and last ratios and more extended response and stable durations $(\mathrm{p}<$ 0.0001, Supplementary Figure 4 and Supplementary Table 4), the Gower distances in the bevacizumab group were not significantly different from the chemotherapy group $(\mathrm{p}=0.06$, 
Figure 3C). This was mainly due to high variability: the lesion-level Gower distances and nadirs varied considerably across organs (Figure 3D, Supplementary Table 5 Table 6), which confounded the patient-level comparison of ILH, as shown in Figure 3C.

Next, we selected the top three metastatic organs for mCRC - liver, lung, and LN - for further analysis. Strikingly, in the bevacizumab arm, the liver lesions had a significantly better response, with lower nadir ratios, last ratios, and Gower distances, as well as a longer response duration than the lesions in the LN and lungs (Figure 3E and Supplementary Table 7). In contrast, chemotherapy alone did not show noticeably better efficacy on the metastases in the liver than those in the $\mathrm{LN}$ and lungs. In the chemotherapy group, the liver lesions appeared to relapse faster than those in the LN, and showed higher heterogeneity than the lung metastases (Figure 3E and Supplementary Table 8). As shown in Figure 3F, compared to the chemotherapy alone, the nadir and last ratios were much lower and the response durations were longer for all metastases in three major organs treated by bevacizumab. The highest efficacy on liver lesions was seen in the bevacizumab arm.

We performed a similar analysis of the panitumumab arm (866 patients), in comparison with the chemotherapy alone arm (FOLFOX or FOLFIRI). Significant benefits in both OS and PFS were observed when adding panitumumab to the chemotherapy (Figure 4A). As in the bevacizumab group, a higher patient-level Gower distance was associated with worse patient survival in the panitumumab group (Figure 4B). Panitumumab showed significantly better efficacy, compared to chemotherapy alone, with much lower tumor nadirs ( $\mathrm{p}<0.0005)$, last ratios ( $\mathrm{p}<0.01)$, and ILH ( $<0.05)$, as well as a more extended response phase ( $<$ 0.0001 ) (Figure 4C and Supplementary Figure 5 and Table 9). The tumor nadir varied across organs, ranging from 0.64 (liver) to 0.92 (other) (Figure 4D and Supplementary Table 10). The lesion response heterogeneity also varied across organs, which further differed between treatments (Figure 4D and Supplementary Table 11). We further compared the response dynamics of the lesions in the three major organs. Similar to bevacizumab, panitumumab exhibited much lower nadir and last ratios and more extended response durations in the selected lesions than chemotherapy alone and had the best response in the liver metastases (Figure 4E, Figure 4F and Supplementary Table 12). The Gower distances of lesions under targeted therapies were significantly lower than chemotherapy in the liver, but a similar observation was not noticed in the lung or lymph nodes metastases (Supplementary Figure 6). Overall, a substantial response heterogeneity across anatomical sites was observed and panitumumab showed remarkably better efficacy on liver metastases than chemotherapy alone.

\section{Lesion response was influenced by patient KRAS status}

Anti-EGFR targeted therapies are prescribed for patients with wild-type $K R A S$. The interaction between $K R A S$ status and lesion response was evaluated in this section. As expected, patients with $K R A S$ mutation $(\mathrm{n}=593)$ had significantly worse OS $(\mathrm{p}<0.0001)$ and PFS ( $\mathrm{p}<0.01)$ compared to the wild-type patients $(\mathrm{n}=765)$, which was consistent with previous findings that $K R A S$ mutations in colon cancers have been associated with poorer survival and increased tumor aggressiveness (25). In the metastases of the three major organs, wild-type patients had relatively lower nadir and last ratios, and more extended 
response durations compared to the $K R A S$ mutant patients ( $\mathrm{p}<0.0001$, Supplementary Figure 7). Since patients with $R A S$ mutations were predicted to have a limited response to panitumumab (26), we further stratified the patients into either with or without panitumumab. The lesion-level comparisons showed that wild-type patients had significantly lower nadir and last ratios in hepatic metastases than their lung and LN metastases. The lesions in the lymph nodes had a nadir ratio of 32\% higher than the lesions in the liver, and lesions in the lung had a nadir ratio of $23 \%$ higher (Figure 5A). The organ-specific response in the KRAS mutant patients was included as a control. In contrast, the liver-favorable effects were not evident in the KRAS mutant patients (Figure 5A and Figure 5B). Panitumumab significantly shrunk the liver metastases, but to a much lesser degree with the lung and LN metastases. The efficacy of panitumumab was higher in the wild-type patients than in the KRAS mutant patients, mostly on hepatic metastases (Figure 5C).

\section{The response of hepatic metastases was closely associated with patient survival}

To compare the survival benefit of organ-specific lesion response, we divided the patients with liver metastases $(n=2,229)$ into subgroups based on whether the shrinkage of the metastases in the liver or other organs was greater than $30 \%$ (the threshold for response in the RECIST v1.1). Patients with responding liver lesions (shrinkage > 30\%) but nonresponding lesions in other organs (shrinkage $\leq 30 \%)$ had significantly better OS $(n=142$, median 546 days) and PFS (median 282 days) than those who had responding lesions in other organs but not in the liver ( $\mathrm{n}=80$, OS 401 days; $\mathrm{n}=79$, PFS 176 days) (Figure 6A).

We also analyzed the patient survival outcomes for those who only had liver and lung metastases $(n=164)$ or liver and LN metastases $(n=329)$. The patients with responding liver lesions but non-responding lung lesions had significantly extended OS ( $\mathrm{n}=27$, median 519 days) than those with responding lung lesions but non-responding liver lesions $(\mathrm{n}=18$, OS 414 days). Similarly, the patients with responding liver lesions but non-responding LN lesions had longer OS ( $\mathrm{n}=57$, median 662 days) than those with responding lung lesions but non-responding liver lesions ( $\mathrm{n}=27$, OS 401 days) (Figure 6B). In contrast, patients with responding LN or lung lesions but non-responding lesions in other organs had a much worse survival than those with other organ lesion shrinkage $>30 \%$ (Supplementary Figure 8A and 8B). These observations strongly suggested the different survival benefits of organ-specific lesion response. We also compared the Gower distance of patients with responding lesions in Figure 6A and Figure 6B. The correlation between the Gower distance and the long-term patient survival was significant at the patient level (Figure 2C and Figure 2D), but we did not observe similar correlations at every organ. It is probably because the long-term patient survival is affected, to different degrees, by the organ-specific responses. Altogether, patient survival was more closely associated with responding lesions in the liver than those in the LN and lungs (Figure 6C).

\section{Discussion}

Our study evaluated the spatiotemporal response heterogeneity (i.e., ILH) across 11,404 metastatic lesions in 2,802 mCRC patients. We characterized this substantial ILH and found that patients with higher ILH (i.e., high Gower distance) had worse survival outcomes. 
Metastases in different anatomical sites responded to the therapies differently. Metastatic lesions in the liver showed better and more uniform response to targeted therapies than lesions in the lungs and LN. Patients with $K R A S$ mutations had less treatment benefits in their liver lesions than the wild-type patients. Regardless of therapy and $K R A S$ status, a favorable response in the liver lesions was more associated with patient survival benefits than lesions at other sites. Our findings provided evidence of the importance of lesion-level response assessments and supported the inclusion of lesion-level responses in the current RECIST approach for improved evaluations of drug efficacy and patient survival outcomes in $\mathrm{mCRC}$.

Several studies observed that the response dynamics across lesions in targeted therapies or immunotherapies were highly heterogeneous and patients who had a lower degree of interlesion heterogeneity had longer survival. $(12,13,18,27)$ We evaluated the spatiotemporal heterogeneity by quantifying the full response dynamics across all metastatic lesions and compared it across treatment types, anatomical sites, and $K R A S$ status. Our study provided a new route to understand intra-tumor heterogeneity, revealing more insights into the prognostic factors for patient survival. Besides, patients with a similar response across lesions are often associated with either a comparable degree of genetic mutations or systemic activations of immune functions, two critical facets for a high response to targeted therapies or immunotherapies, respectively. (5) Our study provided further evidence showing that a uniform response across lesions is highly informative to therapeutic benefit of both chemotherapies and targeted therapies in mCRC. The lesion-level phenotypic response provided us another perspective to understand drug efficacy and resistance. These response differences across metastatic lesions might be attributed to the genetically and epigenetically divergent tumor cell clones within each lesion filtered by the organ-specific tumor immune microenvironment (28). Tumor development has been widely regarded as a process of Darwinian evolution (29). Many metastatic lesions, subject to different organ-specific evolutionary filters, have accumulated multiple genetically distinct sub-clones by the time of diagnosis (30). The clonal heterogeneity across metastatic lesions may result in different levels of sensitivity and resistance to treatments, leading to high phenotypic heterogeneity across metastatic lesions (31). The relatively uniform response in many long-term survivors could be partially due to similar genetic compositions, as well as reduced heterogeneity across metastatic lesions (32). Interestingly, the phenotypic diversity in lesions in the LN did not appear to be significantly higher than in the other distant lesions in our study, even though the genetic diversity was found to be much broader within LN metastases than distant lesions (8). KRAS mutation status showed a much higher impact on the response of liver lesions than lesions at the other sites. The $K R A S$ mutation status in patients could not be taken to infer the $K R A S$ status of all metastatic lesions, concerning the phylogenetic diversity and varying evolutionary selective pressures in each metastasis in colorectal cancers. (8) Although genotype-phenotype mapping was not within the scope of this study, our analyses reinforced the importance of the joint consideration of cancer genotyping with the lesion-level response phenotypes to better understand treatment efficacy and resistance. Overall, the current RECIST approach should be modified to extend beyond the sum of all target lesions, as it may potentially obscure the prognostic values of the unique response dynamics of individual metastases. 
Since the treatment responses varied by anatomical sites, the correlation between the Gower distance and the treatment efficacy did not stand for all organs. We observed a significant decrease of the Gower distance in liver metastases in targeted therapy, but not in the lung and lymph node metastases. (Figure 3E and Figure 4E) The favorable effect of targeted therapies on liver metastatic lesions could be ascribed to the high antibody penetrations in the liver compared to other organs. The liver is a highly perfused organ with sinusoidal vascular structures (33). Bevacizumab uptake in the liver and liver metastases was much higher than into the lungs and LN, according to an ${ }^{89} \mathrm{Zr}$-bevacizumab PET study (34). Similarly, panitumumab also exhibited a greater penetration into the liver than the lungs $(35,36)$. Interestingly, the target expressions for two targeted therapies in liver metastases, VEGF and EGFR, were not higher than the LN and lung metastases in mCRC, indicating that the preferable uptake of antibodies in liver metastases was due to higher drug penetration but not enhanced target expression (37-39).

We observed that a response of liver metastasis greatly accounted for the therapeutic benefit of targeted therapies, indicating patient long-term survival. The liver is generally regarded as an immunologically tolerant organ, in which the hepatic adaptive immune cells become readily tolerogenic, promoting immunosuppressive tumor microenvironment (40). The liver metastasis is critical in the system activations of immune functions. Therefore, the targeted therapies involving the activation of cytotoxic $\mathrm{T}$ cells (such as checkpoint blockades) are often less effective for liver metastases, compared to metastases in other anatomical sites $(18,27)$. The favorable response in liver metastasis unfortunately vanished in $K R A S$ mutant patients. Unlike checkpoint blockades, the primary tumor-restraining mechanism of bevacizumab and panitumumab is to block or neutralize their respective antigens rather than eliciting effector functions (41). Different mechanisms of action helped explain why the targeted therapies evaluated in this study showed favorable effects on liver lesions compared to other organs. Intriguingly, the favorable benefit of panitumumab on liver lesions was blunted in patient with the $K R A S$ mutation, suggesting the interplay between drug pharmacology and tumor biology. More investigations are warranted to validate this perspective.

The liver is the most common metastatic site for CRC due to its unique location in proximity to the colorectal system. Approximately 15\% of CRC patients have synchronous hepatic metastases at the time of diagnosis, and another 50\% will develop liver metastases in the process of treatment (42). There is a clear survival benefit in first performing a surgical resection of liver metastases when feasible, compared to resecting primary tumors first (43). One rationale behind the liver-first strategy is that liver metastasis is the main lethal factor in CRC patients. Extensive tumor hypoxia has been observed in liver metastases of CRC (44), and the hypoxic status of solid tumors is related to poor prognosis (45). The five-year survival rate is only $6 \%$ for patients with liver metastases. The early resection or reduction of liver lesions mitigate the risks of their growing beyond respectability (46). Our results were in line with the liver-first strategy and indicated the relatively higher survival benefit of favorable responses in liver metastases than in those of other organs, providing an incentive for liver-first management. For those who were not qualified for liver lesion resections, targeted therapies, such as bevacizumab or panitumumab, should be prioritized due to their favorable efficacy in liver metastases. 
Our study has limitations. We analyzed data from multiple Phase III trials that had discrepancies in clinical trial design, treatment duration, and lesion evaluation frequency, resulting in high variabilities in the analyzed metrics that challenged the statistical robustness. These trials were conducted between 2002 and 2013, which made our analyses fail to reveal the current better surgical, medical, and supportive care for cancer patients. In addition, our data did not include cetuximab, another broadly prescribed targeted therapy for mCRC. Cetuximab can elicit immunologic antitumor effects, such as antibody-dependent cellular cytotoxicity, thus differing from panitumumab (47). The favorable efficacy in liver metastases by panitumumab may not be the case by cetuximab. Thus, our findings related to targeted therapy should be interpreted within the two included antibodies. Moreover, our study only analyzed the target lesions and did not include non-target lesions and new lesions, which are also critical for assessing treatment response and patient survival (9).

Furthermore, the Gower distance is a metric primarily to reveal the heterogeneity of response across lesions, not yet as a powerful predictor of clinical responses. To take the Gower distance as a predictor of patient long-term survival, we should have more analyses and validations on the lead time and the predictive specificity/accuracy, which is beyond the scope of our analysis.

In conclusion, we identified the high spatiotemporal response heterogeneity across metastatic lesions in mCRC patients. The patients with higher ILH tended to have worse survival. Targeted therapies, in combination with the standard chemotherapies, showed favorable efficacy in liver metastases than chemotherapy alone, which was blunted for patients with KRAS mutation. Such favorable responses in the liver are more closely associated with patient long-term survival than metastatic lesions in other anatomical sites. These findings strongly support the importance of evaluating lesion-level responses for the improved assessment of drug efficacy and patient survival.

\title{
Supplementary Material
}

Refer to Web version on PubMed Central for supplementary material.

\section{Acknowledgements}

This work was supported by NIH grant GM119661.

Financial support: Y. Cao and J. Zhou were funded by National Institute of Health (GM119661).

\author{
Abbreviations \\ RECIST \\ LN \\ GI \\ CRC/mCRC \\ PFS \\ Response Evaluation Criteria in Solid Tumors \\ lymph nodes \\ gastrointestinal \\ colorectal cancer/metastatic colorectal cancer \\ progression-free survival
}

Cancer Res. Author manuscript; available in PMC 2021 November 01. 


\begin{tabular}{|c|c|}
\hline OS & overall survival \\
\hline PPS & post-progression survival \\
\hline ILH & inter-lesion heterogeneity \\
\hline Beva & bevacizumab \\
\hline Pani & panitumumab \\
\hline Chemo & chemotherapy \\
\hline FOLFOX & $\begin{array}{l}\text { folinic acid (leucovorin), fluorouracil (5-FU), and oxaliplatin } \\
\text { (Eloxatin) }\end{array}$ \\
\hline FOLFIRI & $\begin{array}{l}\text { folinic acid (leucovorin), fluorouracil (5-FU), and irinotecan } \\
\text { (Camptosar) }\end{array}$ \\
\hline МАРК & mitogen-activated protein kinase \\
\hline EGFR & epidermal growth factor receptor \\
\hline VEGF & vascular endothelial growth factor \\
\hline BSA & body surface area \\
\hline LDH & lactate dehydrogenase \\
\hline HR & hazard ratio \\
\hline
\end{tabular}

\section{References}

1. Welch DR, Hurst DR. Defining the hallmarks of metastasis. Cancer Res. 2019;79:3011-27. [PubMed: 31053634]

2. Lambert AW, Pattabiraman DR, Weinberg RA. Emerging biological principles of metastasis. Cell. 2017;168:670-91. [PubMed: 28187288]

3. Angelova M, Mlecnik B, Vasaturo A, Bindea G, Fredriksen T, Lafontaine L, et al. Evolution of metastases in space and time under immune selection. Cell. 2018;175:751-65. [PubMed: 30318143]

4. Huang Q, Hu X, He W, Zhao Y, Hao S, Wu Q, et al. Fluid shear stress and tumor metastasis. Am J Cancer Res. 2018;8:763. [PubMed: 29888101]

5. Russo M, Siravegna G, Blaszkowsky LS, Corti G, Crisafulli G, Ahronian LG, et al. Tumor heterogeneity and lesion-specific response to targeted therapy in colorectal cancer. Cancer Discov. 2016;6:147-53. [PubMed: 26644315]

6. Khan KH, Cunningham D, Werner B, Vlachogiannis G, Spiteri I, Heide T, et al. Longitudinal liquid biopsy and mathematical modeling of clonal evolution forecast time to treatment failure in the PROSPECT-C phase II colorectal cancer clinical trial. Cancer Discov. 2018;8:1270-85. [PubMed: 30166348]

7. Naxerova K, Reiter JG, Brachtel E, Lennerz JK, Van De Wetering M, Rowan A, et al. Origins of lymphatic and distant metastases in human colorectal cancer. Science (80-). 2017;357:55-60.

8. Reiter JG, Hung W-T, Lee I-H, Nagpal S, Giunta P, Degner S, et al. Lymph node metastases develop through a wider evolutionary bottleneck than distant metastases. Nat Genet. 2020;1-9. [PubMed: 31911675]

9. Eisenhauer EA, Therasse P, Bogaerts J, Schwartz LH, Sargent D, Ford R, et al. New response evaluation criteria in solid tumours: revised RECIST guideline (version 1.1). Eur J Cancer. 2009;45:228-47. [PubMed: 19097774] 
10. Nishino M Tumor response assessment for precision cancer therapy: response evaluation criteria in solid tumors and beyond. Am Soc Clin Oncol Educ B. 2018;38:1019-29.

11. Siravegna G, Lazzari L, Crisafulli G, Sartore-Bianchi A, Mussolin B, Cassingena A, et al. Radiologic and genomic evolution of individual metastases during HER2 blockade in colorectal cancer. Cancer Cell. 2018;34:148-62. [PubMed: 29990497]

12. Schmid S, Diem S, Li Q, Krapf M, Flatz L, Leschka S, et al. Organ-specific response to nivolumab in patients with non-small cell lung cancer (NSCLC). Cancer Immunol Immunother. 2018;67:1825-32. [PubMed: 30171269]

13. Menzies AM, Haydu LE, Carlino MS, Azer MWF, Carr PJA, Kefford RF, et al. Inter-and intrapatient heterogeneity of response and progression to targeted therapy in metastatic melanoma. PLoS One. 2014;9:e85004. [PubMed: 24400126]

14. Parker NR, Hudson AL, Khong P, Parkinson JF, Dwight T, Ikin RJ, et al. Intratumoral heterogeneity identified at the epigenetic, genetic and transcriptional level in glioblastoma. Sci Rep. 2016;6:22477. [PubMed: 26940435]

15. Kawachi H, Tamiya M, Tamiya A, Ishii S, Hirano K, Matsumoto H, et al. Association between metastatic sites and first-line pembrolizumab treatment outcome for advanced non-small cell lung cancer with high PD-L1 expression: a retrospective multicenter cohort study. Invest New Drugs. 2020;38:211-8. [PubMed: 31784866]

16. Beaumont H, Faye N, Iannessi A, Chamorey E, Kliffa C, Hsieh C-Y. Differences in Sensitivity to New Therapies Between Primary and Metastatic Breast Cancer: A Need to Stratify the Tumor Response? 2020;

17. Yang K, Li J, Bai C, Sun Z, Zhao L. Efficacy of immune checkpoint inhibitors in non-small-cell lung cancer patients with different metastatic sites: a systematic review and meta-analysis. Front Oncol. 2020;10:1098. [PubMed: 32733805]

18. Osorio JC, Arbour KC, Le DT, Durham JN, Plodkowski AJ, Halpenny DF, et al. Lesion-level response dynamics to programmed cell death protein (PD-1) blockade. J Clin Oncol. 2019;37:3546-55. [PubMed: 31675272]

19. Siegel RL, Miller KD, Goding Sauer A, Fedewa SA, Butterly LF, Anderson JC, et al. Colorectal cancer statistics, 2020. CA Cancer J Clin. 2020;

20. Siegel RL, Miller KD, Jemal A. Cancer statistics, 2015. CA Cancer J Clin. 2015;65:5. [PubMed: 25559415]

21. Kirstein MM, Lange A, Prenzler A, Manns MP, Kubicka S, Vogel A. Targeted therapies in metastatic colorectal cancer: a systematic review and assessment of currently available data. Oncologist. 2014;19:1156. [PubMed: 25326159]

22. Gower JC. A general coefficient of similarity and some of its properties. Biometrics. 1971;857-71.

23. McDonald K-A, Kawaguchi T, Qi Q, Peng X, Asaoka M, Young J, et al. Tumor heterogeneity correlates with less immune response and worse survival in breast cancer patients. Ann Surg Oncol. 2019;26:2191-9. [PubMed: 30963401]

24. Vera-Yunca D, Girard P, Parra-Guillen ZP, Munafo A, Trocóniz IF, Terranova N. Machine Learning Analysis of Individual Tumor Lesions in Four Metastatic Colorectal Cancer Clinical Studies: Linking Tumor Heterogeneity to Overall Survival. AAPS J. 2020;22:1-12.

25. Arrington AK, Heinrich EL, Lee W, Duldulao M, Patel S, Sanchez J, et al. Prognostic and predictive roles of KRAS mutation in colorectal cancer. Int J Mol Sci. 2012;13:12153-68. [PubMed: 23202889]

26. Douillard J-Y, Oliner KS, Siena S, Tabernero J, Burkes R, Barugel M, et al. PanitumumabFOLFOX4 treatment and RAS mutations in colorectal cancer. N Engl J Med. 2013;369:1023-34. [PubMed: 24024839]

27. Pires da Silva I, Lo S, Quek C, Gonzalez M, Carlino MS, Long GV, et al. Site-specific response patterns, pseudoprogression, and acquired resistance in patients with melanoma treated with ipilimumab combined with anti-PD-1 therapy. Cancer. 2020;126:86-97. [PubMed: 31584722]

28. Hunter KW, Amin R, Deasy S, Ha N-H, Wakefield L. Genetic insights into the morass of metastatic heterogeneity. Nat Rev Cancer. 2018;18:211. [PubMed: 29422598]

29. Greaves M Evolutionary determinants of cancer. Cancer Discov. 2015;5:806-20. [PubMed: 26193902] 
30. McGranahan N, Swanton C. Clonal heterogeneity and tumor evolution: past, present, and the future. Cell. 2017;168:613-28. [PubMed: 28187284]

31. Sanborn JZ, Chung J, Purdom E, Wang NJ, Kakavand H, Wilmott JS, et al. Phylogenetic analyses of melanoma reveal complex patterns of metastatic dissemination. Proc Natl Acad Sci. 2015;112:10995-1000. [PubMed: 26286987]

32. Burrell RA, McGranahan N, Bartek J, Swanton C. The causes and consequences of genetic heterogeneity in cancer evolution. Nature. 2013;501:338-45. [PubMed: 24048066]

33. Lautt WW. Hepatic circulation: physiology and pathophysiology. Colloq Ser Integr Syst Physiol from Mol to Funct. 2009. page 1-174.

34. Oosting SF, Brouwers AH, van Es SC, Nagengast WB, Munnink THO, Lub-de Hooge MN, et al. 89Zr-bevacizumab PET visualizes heterogeneous tracer accumulation in tumor lesions of renal cell carcinoma patients and differential effects of antiangiogenic treatment. J Nucl Med. 2015;56:63-9. [PubMed: 25476536]

35. Wong KJ, Baidoo KE, Nayak TK, Garmestani K, Brechbiel MW, Milenic DE. In vitro and in vivo pre-clinical analysis of a F (ab') 2 fragment of panitumumab for molecular imaging and therapy of HER1-positive cancers. EJNMMI Res. 2011;1:1.

36. Liu Z, Liu Y, Jia B, Zhao H, Jin X, Li F, et al. Epidermal growth factor receptor-targeted radioimmunotherapy of human head and neck cancer xenografts using 90Y-labeled fully human antibody panitumumab. Mol Cancer Ther. 2010;9:2297-308. [PubMed: 20682654]

37. Duff SE, Jeziorska M, Rosa DD, Kumar S, Haboubi N, Sherlock D, et al. Vascular endothelial growth factors and receptors in colorectal cancer: implications for anti-angiogenic therapy. Eur $\mathbf{J}$ Cancer. 2006;42:112-7. [PubMed: 16321517]

38. Cascinu S, Graziano F, Catalano V, Staccioli MP, Barni S, Giordani P, et al. Differences of vascular endothelial growth factor (VEGF) expression between liver and abdominal metastases from colon cancer. Implications for the treatment with VEGF inhibitors. Clin Exp Metastasis. 2000;18:651-5. [PubMed: 11827068]

39. Scartozzi M, Bearzi I, Berardi R, Mandolesi A, Fabris G, Cascinu S. Epidermal growth factor receptor (EGFR) status in primary colorectal tumors does not correlate with EGFR expression in related metastatic sites: implications for treatment with EGFR-targeted monoclonal antibodies. $\mathrm{J}$ Clin Oncol. 2004;22:4772-8. [PubMed: 15570078]

40. Zheng M, Tian Z. Liver-mediated adaptive immune tolerance. Front Immunol. 2019;10:2525. [PubMed: 31787967]

41. García-Foncillas J, Sunakawa Y, Aderka D, Wainberg Z, Ronga P, Witzler P, et al. Distinguishing features of cetuximab and panitumumab in colorectal cancer and other solid tumors. Front Oncol. 2019;9:849. [PubMed: 31616627]

42. Zarour LR, Anand S, Billingsley KG, Bisson WH, Cercek A, Clarke MF, et al. Colorectal cancer liver metastasis: evolving paradigms and future directions. Cell Mol Gastroenterol Hepatol. 2017;3:163-73. [PubMed: 28275683]

43. Manfredi S, Lepage C, Hatem C, Coatmeur O, Faivre J, Bouvier A-M. Epidemiology and management of liver metastases from colorectal cancer. Ann Surg. 2006;244:254. [PubMed: 16858188]

44. van Laarhoven HWM, Kaanders JHAM, Lok J, Peeters WJM, Rijken PFJW, Wiering B, et al. Hypoxia in relation to vasculature and proliferation in liver metastases in patients with colorectal cancer. Int J Radiat Oncol Biol Phys. 2006;64:473-82. [PubMed: 16242253]

45. Vaupel P, Mayer A, Höckel M. Tumor hypoxia and malignant progression. Methods Enzymol. 2004. page 335-54. [PubMed: 15063685]

46. Siriwardena AK, Mason JM, Mullamitha S, Hancock HC, Jegatheeswaran S. Management of colorectal cancer presenting with synchronous liver metastases. Nat Rev Clin Oncol. 2014;11:446. [PubMed: 24889770]

47. Srivastava RM, Lee SC, Andrade Filho PA, Lord CA, Jie H-B, Davidson HC, et al. Cetuximabactivated natural killer and dendritic cells collaborate to trigger tumor antigen-specific T-cell immunity in head and neck cancer patients. Clin Cancer Res. 2013;19:1858-72. [PubMed: 23444227] 


\section{Statement of Significance}

Findings support the modification of RECIST criteria to include individual lesion response to improve assessments of drug efficacy. 
A
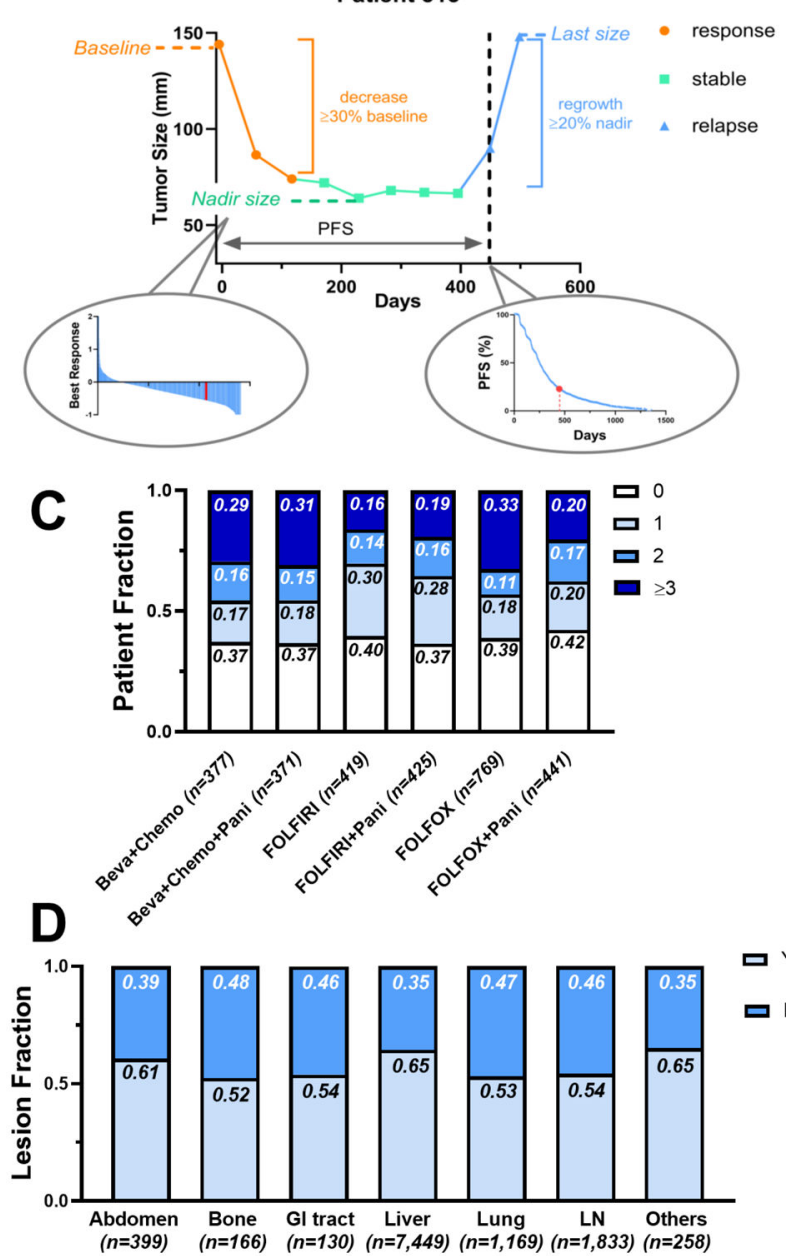

B
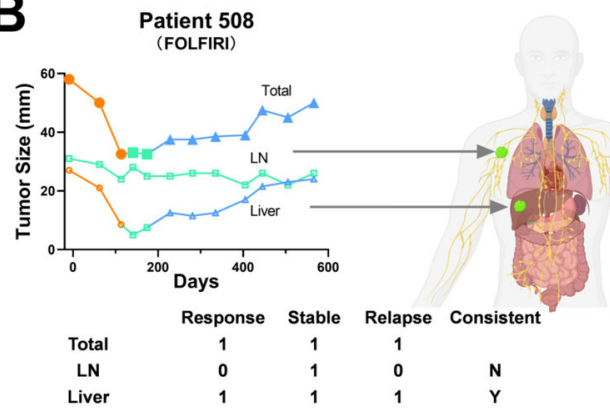

Patient 501 (FOLFIRI+Pani)
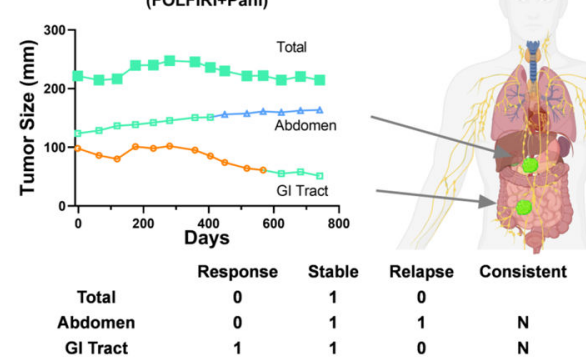

Figure 1. Heterogeneous response dynamics across metastatic lesions.

(A) Five variables (response, stable, and relapse phase, tumor nadir size, and last size before the end of trials normalized by the baseline) were extracted to describe tumor response patterns. The left sub-panel is the nadir waterfall plot and the right sub-panel is the PFS Kaplan-Meier curve of all the patients in the dataset. The nadir and PFS of patient 513 were labeled in red in both sub-panels. (B) Total tumor burden and individual metastatic lesion response dynamics during the treatment in three representative patients, patient 508, 501, and 1312. The lesion response, stable and relapse phases are labeled in orange, green, and blue respectively. Three binary variables (Response, Stable, Relapse) represent whether their corresponding phases exist $(=1)$ or not $(=0)$. Lesions were defined as inconsistent $(\mathrm{N})$ if their phase variables were not consistent with those in the total tumor burden. Otherwise, they would be defined as consistent (Y). (C) The fractions of patients with $0,1,2$, or $\geq 3$ inconsistent metastatic lesions across treatments. (D) The fractions of lesions inconsistent $(\mathrm{N})$ or consistent $(\mathrm{Y})$ with the total tumor burden across the anatomical sites. 
A

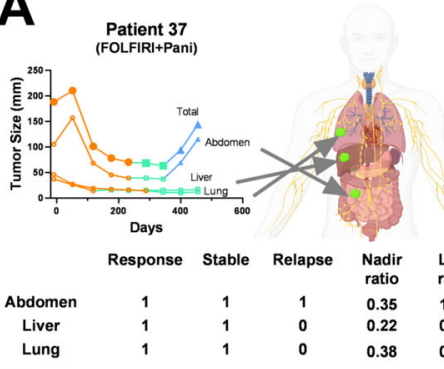

B

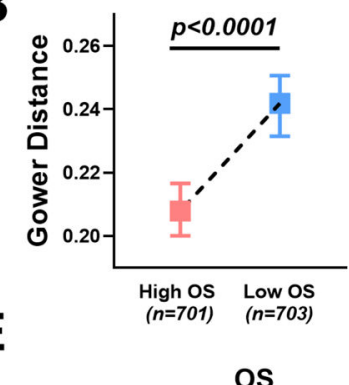

OS

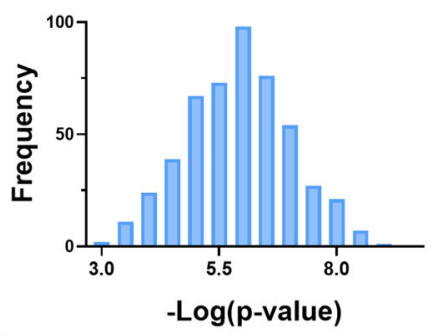

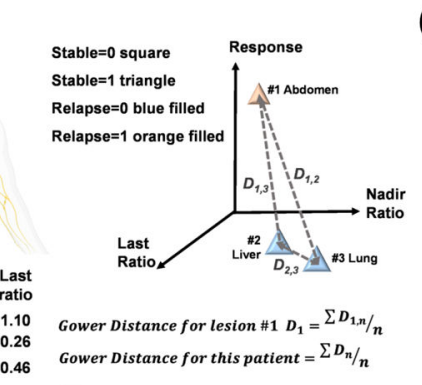

D
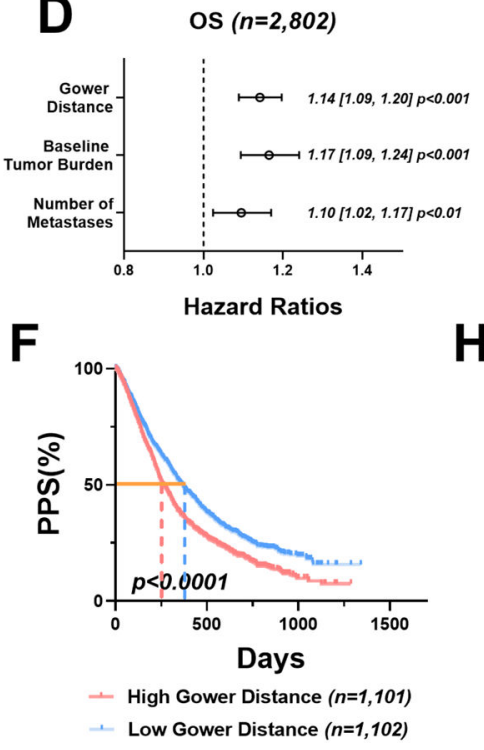

$p<0.0001$

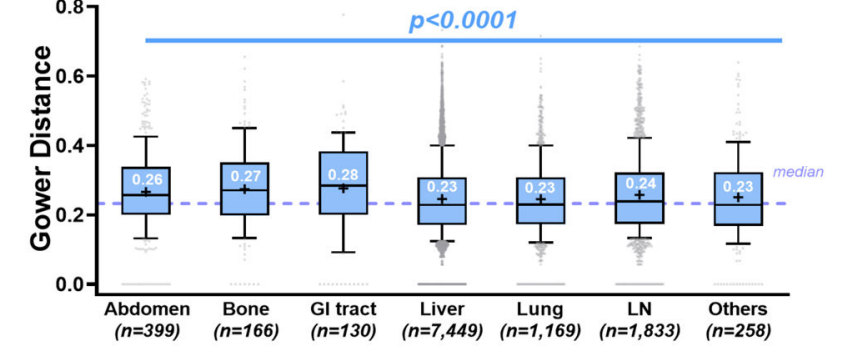

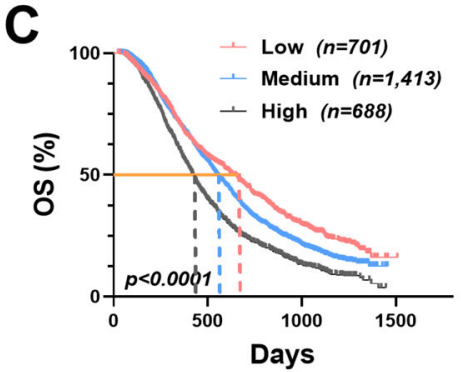

PFS $(n=2,774)$

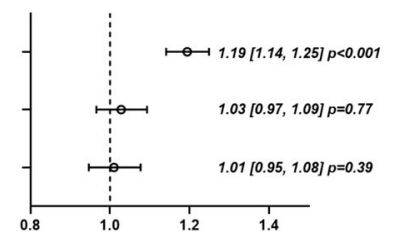

H

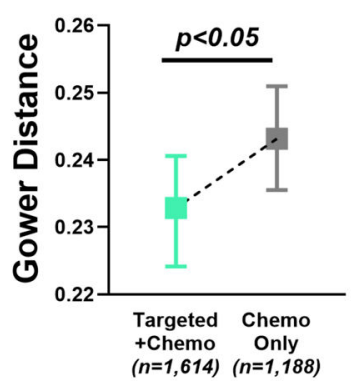

Figure 2. Response heterogeneity across metastatic lesions is associated with survival.

(A) Five parameters extracted from lesion response patterns, including three phase variables (response, stable, and relapse), nadir ratio (baseline-normalized nadir), and last ratio (baseline-normalized last size), were used to calculate the Gower distance. (B) Median with 95\% confidence interval of the Gower distances in patients with top 25\% overall survival (high OS) versus patients with bottom 25\% overall survival (low OS). (C) Kaplan-Meier curves of patient OS grouped by Gower distance ( $25^{\text {th }}$ and $75^{\text {th }}$ quantiles as the cut-offs). (D) Forest plots of the Cox proportional hazards model stratified by treatment type on the OS and PFS, controlling for baseline tumor burden and number of metastases. The hazard ratio with a 95\% confidence interval and the p value of each covariate is labeled in the plot. (E) The $\mathrm{p}$ value distributions of the Gower distance in the OS Cox proportional model on the synthesized datasets (randomly select $80 \%$ of the original dataset). (F) Kaplan-Meier curves of patient post-progression survival (PPS) grouped by high vs low Gower distance (median 
as the cut-off). (G) Boxplot of lesion-level Gower distances grouped by anatomical site. The dashed purple line represents the median of the Gower distances. The box extends from the $25^{\text {th }}$ to $75^{\text {th }}$ percentiles and the line in the middle is plotted as the median (labeled in white). The mean of the group is plotted as the symbol "+". The whiskers are drawn down to the $10^{\text {th }}$ percentile and up to the $90^{\text {th }}$ percentile. Points below and above the whiskers are drawn as gray individual points. (H) Median with $95 \%$ confidence interval of patient-level Gower distances in targeted therapy combined with chemotherapy (Targeted + Chemo) versus chemotherapy alone (Chemo Alone). 


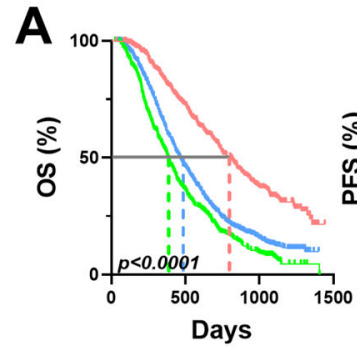

D $\square$ Beva+Chemo $(n=1,634)$
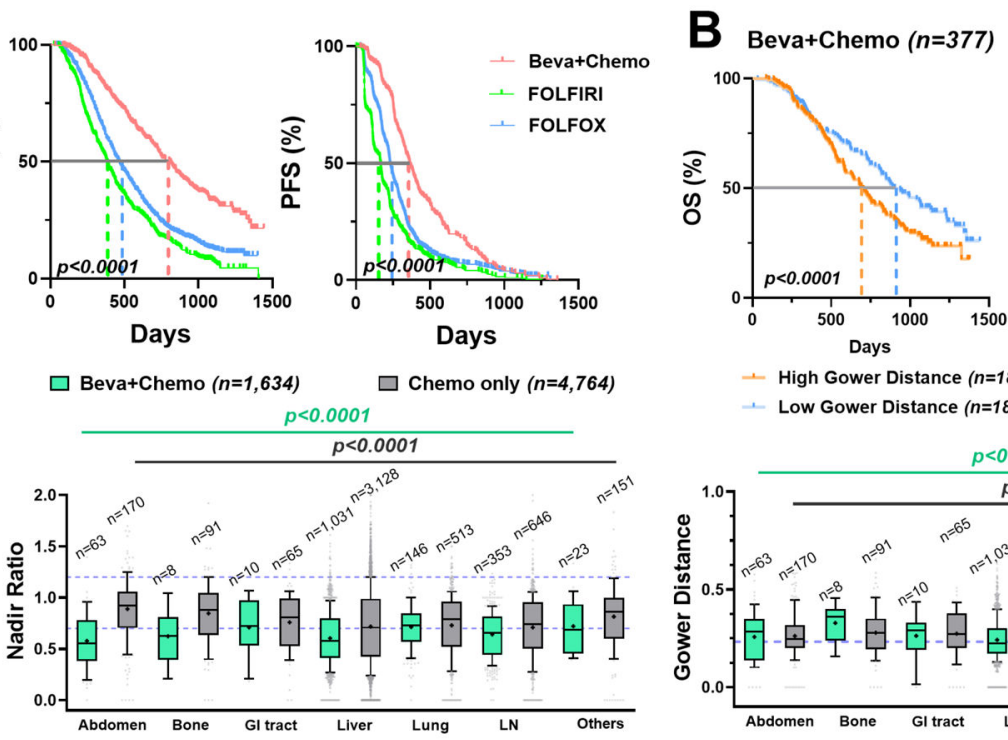

- High Gower Distance ( $n=188)$

+ Low Gower Distance ( $n=189)$

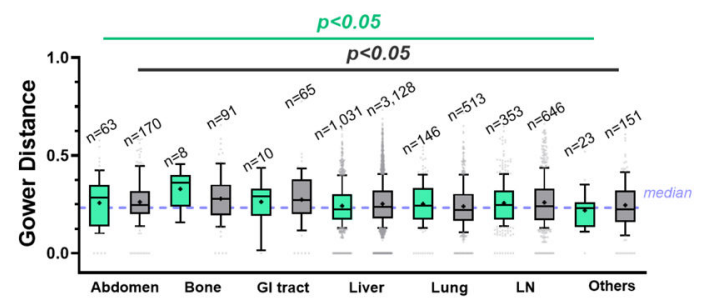

E Beva+Chemo $(n=1,530) \quad$ Chemo Only $(n=4,287)$
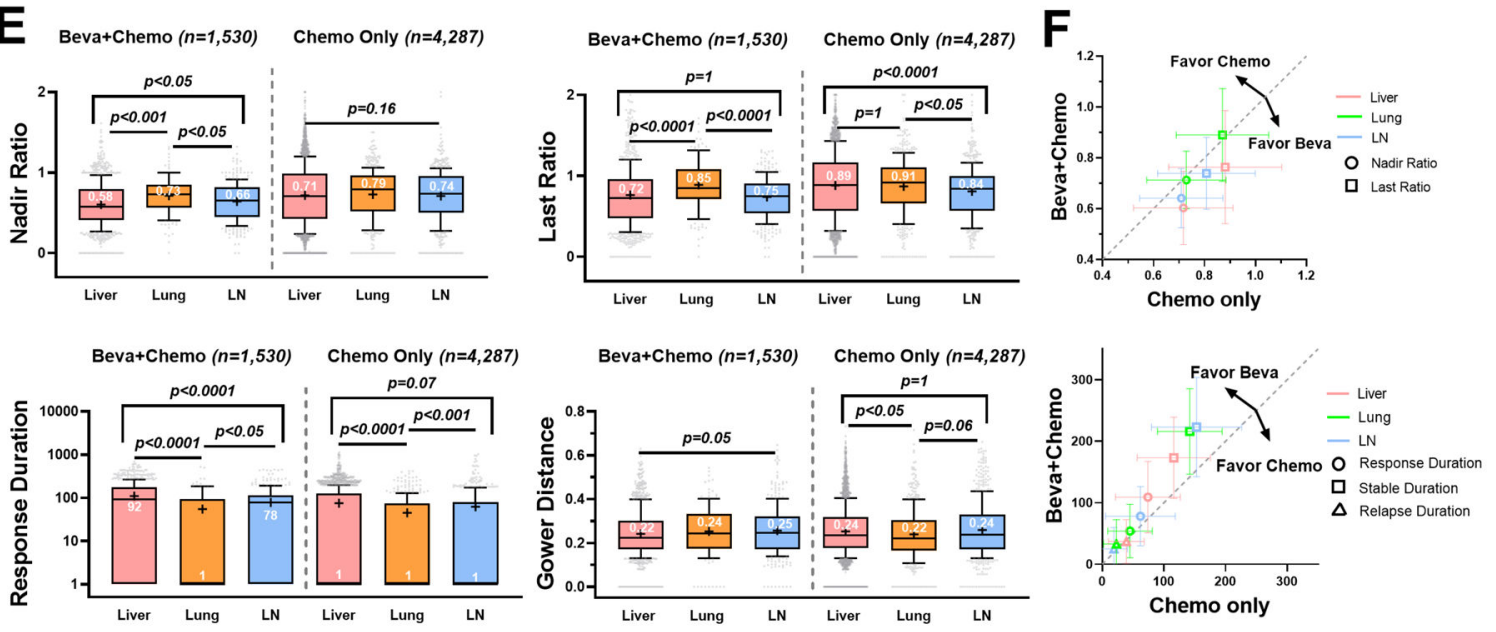

Figure 3. Bevacizumab reduced lesion response heterogeneity and exhibited favorable efficacy on hepatic metastases.

Beva+Chemo: bevacizumab plus chemotherapy; Chemo: chemotherapy alone. (A) KaplanMeier curves of the OS (left) and PFS (right) in Beva + Chemo ( $n=377)$, FOLFOLX ( $n=$ 769 in OS and $n=741$ in PFS) and FOLFIRI $(n=419)$ group. (B) Kaplan-Meier curves of the OS grouped by Gower distance (median as the cut-off) in the Beva + Chemo group. (C) Median with $95 \%$ confidence interval of the patient-level Gower distances in Beva + Chemo versus Chemo (FOLFOX and FOLFIRI) group. (D) Boxplots of the lesions' nadir ratios with the 0.7 and 1.2 values labeled in purple dashed line (left) and the lesions' Gower distances with median labeled in purple dashed line (right) across anatomical sites. (E) Boxplots of the nadir ratio, last ratio, response duration, and Gower distance in liver ( $\mathrm{n}=$ $1,031)$, lung $(n=146)$ and LN metastases $(n=353)$ in the Beva + Chemo (left) compared to the Chemo only (right, liver $n=3,128$; lung $n=513 ; \mathrm{LN} n=646$ ). The durations were all added with one to avoid zero values in logarithmic plots. (F) The mean $\pm 50 \%$ standard deviation of nadir ratio and last ratio (upper) and response, stable, and relapse durations (lower) in the Beva + Chemo versus the Chemo only group. The dashed gray line is $\mathrm{y}=\mathrm{x}$. 

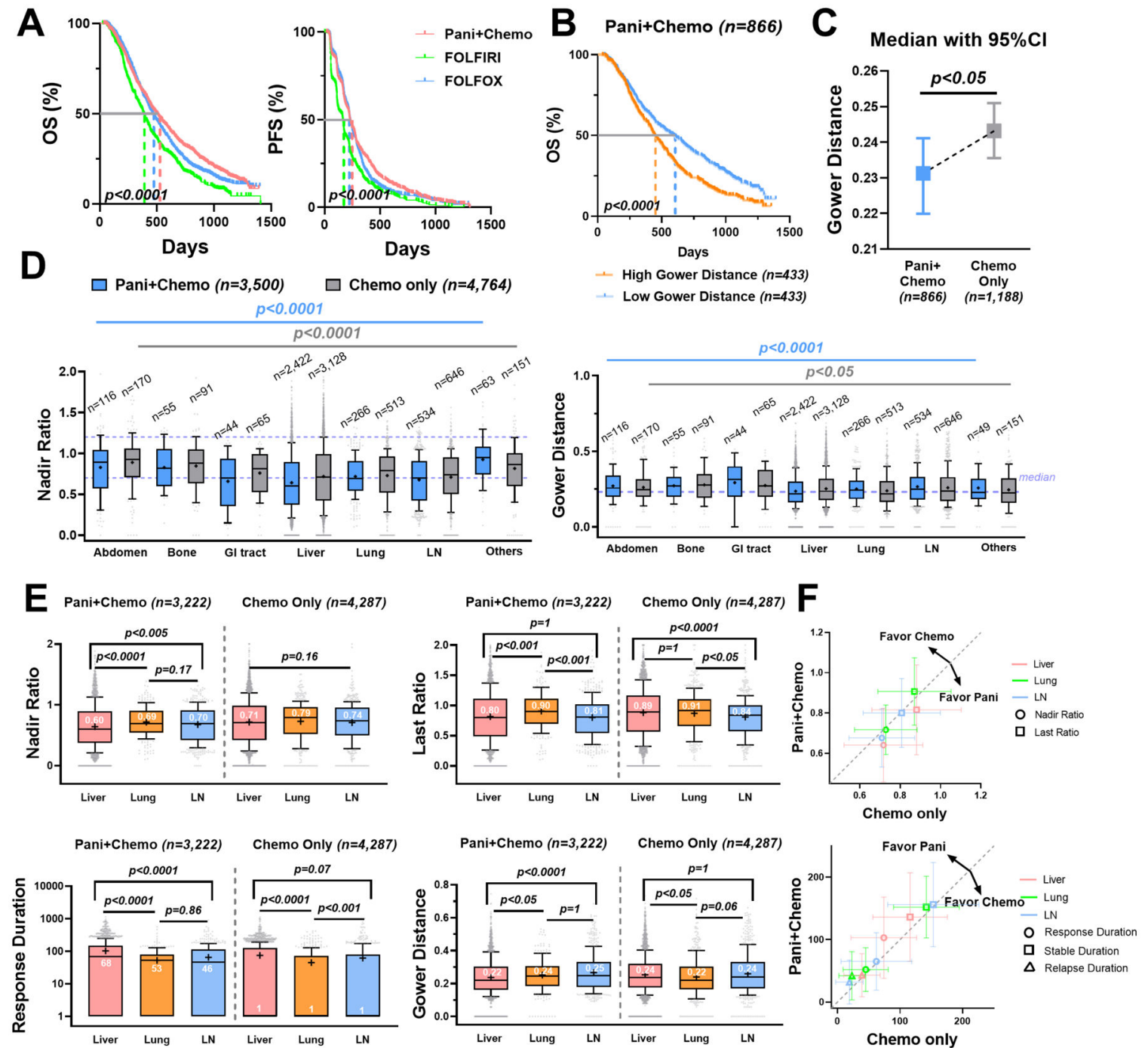

Figure 4. Panitumumab reduced lesion response heterogeneity and showed favorable efficacy on liver metastases.

Pani + Chemo: panitumumab plus chemotherapy; Chemo: chemotherapy alone. (A) KaplanMeier curves of the OS (left) and the PFS (right) in the Pani + Chemo ( $n=866)$, FOLFOLX ( $\mathrm{n}=769$ in OS, $\mathrm{n}=741$ in PFS) and FOLFIRI $(\mathrm{n}=419)$ group. (B) Kaplan-Meier curves of the OS grouped by the Gower distance (median as the cut-off) in the Pani + Chemo group. (C) Median with 95\% confidence interval of the patient-level Gower distances in Pani + Chemo versus Chemo (FOLFOX and FOLFIRI) group. (D) Boxplots of the lesions' nadir ratios with the 0.7 and 1.2 values labeled in purple dashed line (left) and the lesions' Gower distances with median labeled in purple dashed line (right) across anatomical sites. (E) Boxplots of the nadir ratio, last ratio, response duration, and Gower distance in liver ( $\mathrm{n}=$ $2,422)$, lungs $(n=266)$ and $L N$ metastases $(n=534)$ in the Pani + Chemo group (left) compared to the Chemo group (right, liver $n=3,128$; lung $n=513$; $L N n=646$ ). (F) The mean $\pm 50 \%$ standard deviation of nadir ratio and last ratio (upper) and response, stable, and relapse durations (lower) in the Pani + Chemo versus Chemo only group. The dashed gray line is $\mathrm{y}=\mathrm{x}$. 


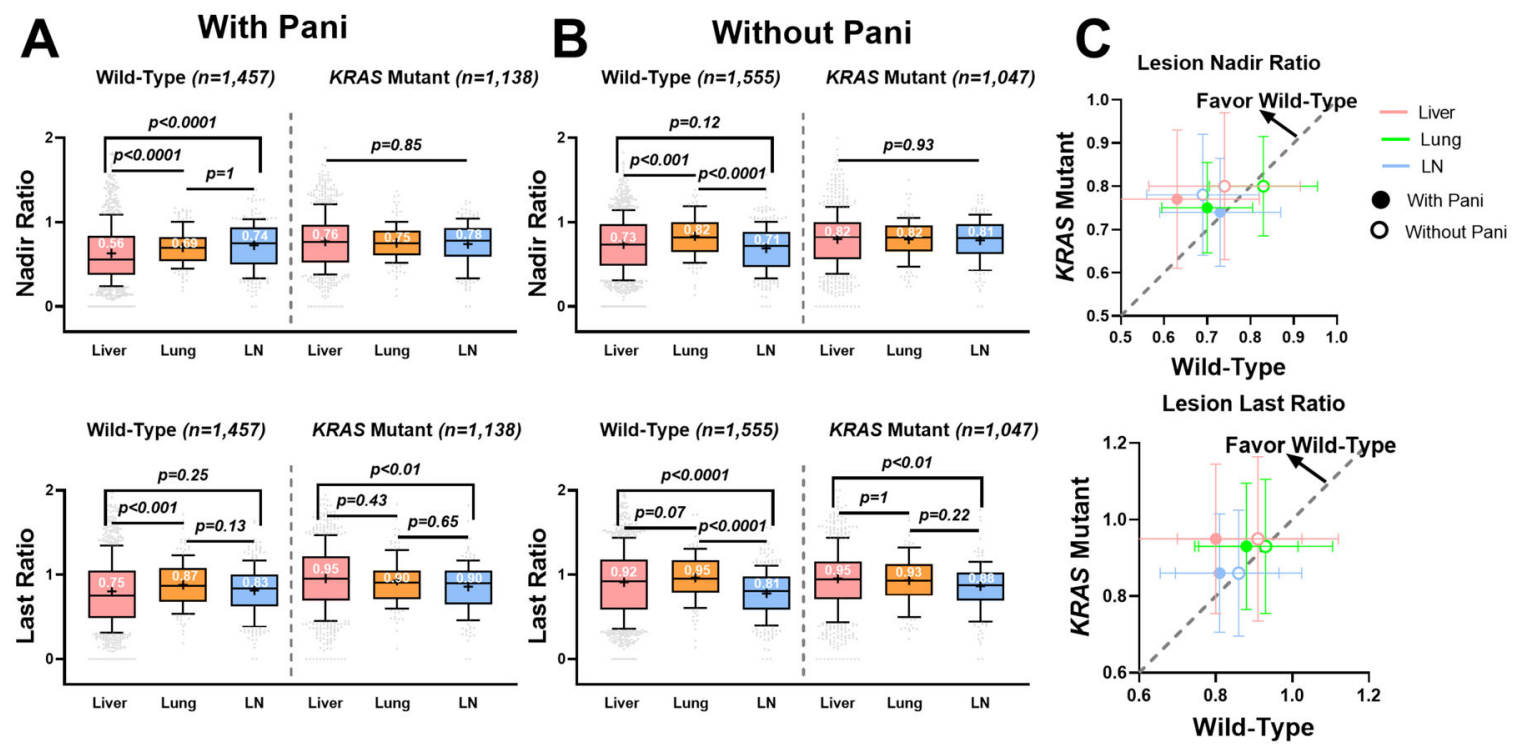

Figure 5. Lesion response heterogeneity was influenced by patient KRAS mutation status. (A) Boxplots of the nadir ratio (upper) and last ratio (lower) of the lesions in the wild-type versus KRAS mutant patients under panitumumab treatment (Beva + Chemo + Pani or FOLFIRI + Pani). (B) Boxplots of the nadir ratio (upper) and last ratio (lower) of the lesions in the wild-type versus KRAS mutant patients without panitumumab treatment (Beva + Chemo or FOLFIRI). (C) The mean $\pm 50 \%$ standard deviation of the nadir ratio (upper) and last ratio (lower) in the wild-type versus $K R A S$ mutant patients. The dashed gray line is y = $\mathrm{x}$. 
A Patients with Liver Metastases $(n=2,229)$

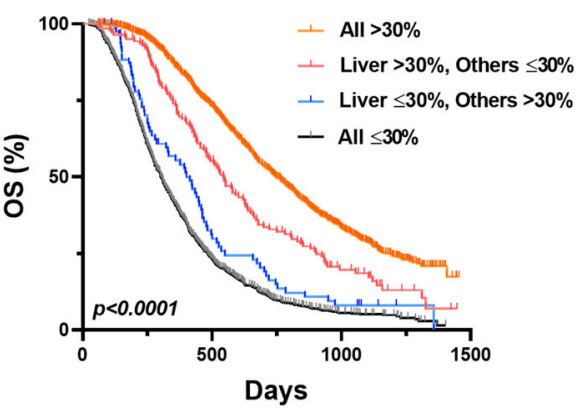

B Patients with liver and lung metastasis $(n=164)$

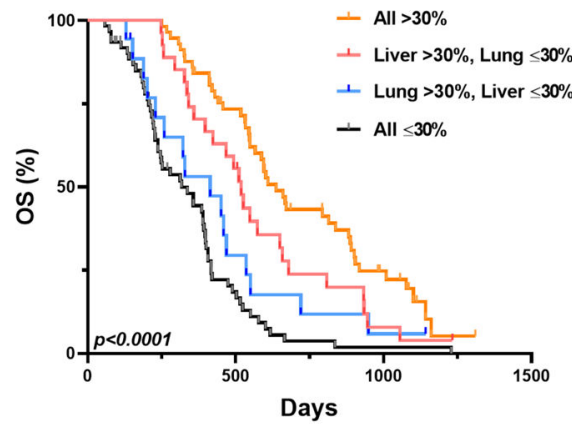

Patients with Liver Metastases $(n=2,203)$

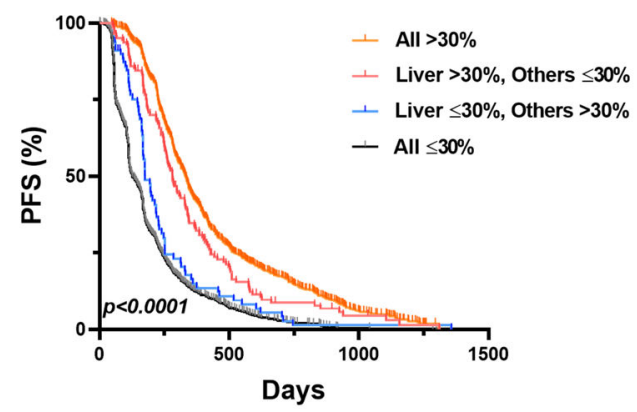

Patients with liver and LN metastasis $(n=329)$

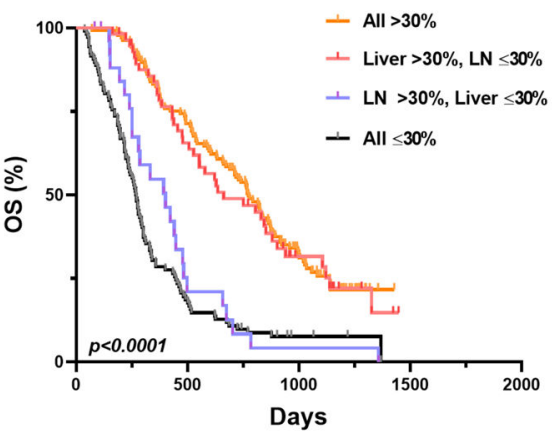

C

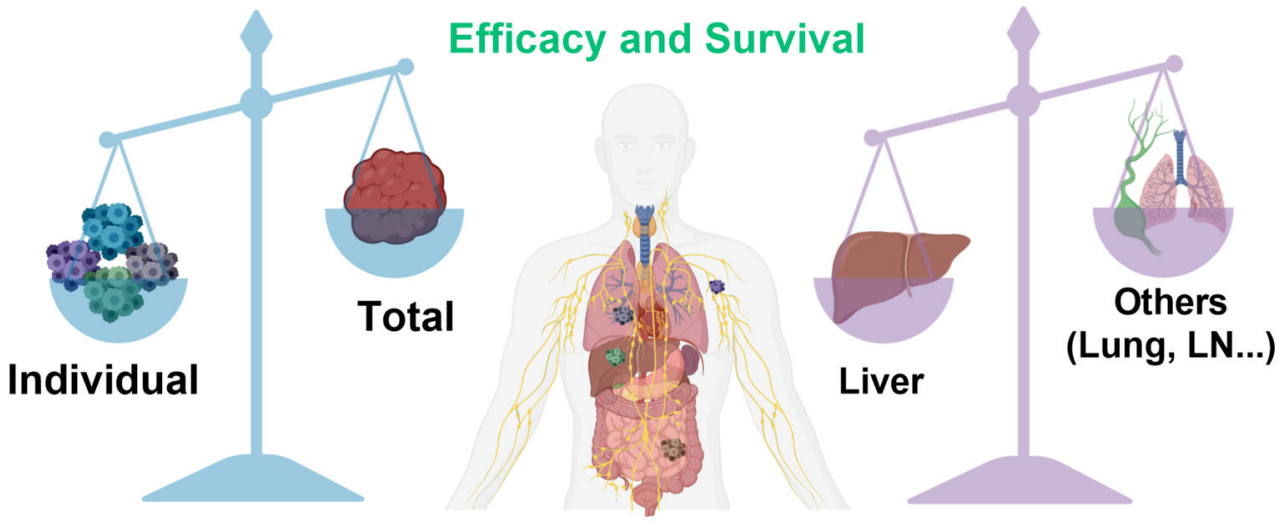

Figure 6. The response of liver lesions was more closely associated with patient survival. (A) Kaplan-Meier curves of the OS (left, $n=2,229$ ) and the PFS (right, $n=2,203$ ) in patients with liver metastases. The patients were divided into four sub-groups: all lesions shrinkage greater than $30 \%$ threshold (all $>30 \%, \mathrm{n}=1,113$ in OS, $\mathrm{n}=1,101$ in PFS); liver lesions shrinkage $>30 \%$ while other lesions shrinkage $\leq 30 \%$ (liver $>30 \%$, others $\leq 30 \%$; $n$ $=142$ ); liver lesions shrinkage $\leq 30 \%$ while other lesions shrinkage $>30 \%$ (liver $\leq 30 \%$, others $>30 \% ; \mathrm{n}=80$ in OS, $\mathrm{n}=79$ in PFS); all lesions shrinkage $\leq 30 \%$ (all $\leq 30 \%, \mathrm{n}=894$ in OS, $n=881$ in PFS). (B) Kaplan-Meier curves of the OS in the patients with liver and lungs metastases only (left; $n=164$ ), or the patients with liver and LN metastases only (right; $\mathrm{n}=329$ ). Similarly, patients were divided into four groups by the lesion shrinkage. In the left panel, all > 30\% ( $\mathrm{n}=57)$; liver $>30 \%$; lung $\leq 30 \%(\mathrm{n}=27)$; lung $>30 \%$, liver $\leq 30 \%$ $(\mathrm{n}=18) ;$ all $\leq 30 \%(\mathrm{n}=62)$. In the right panel, all $>30 \%(\mathrm{n}=138)$; liver $>30 \%$; $\mathrm{LN} \leq 30 \%$ $(\mathrm{n}=57)$; $\mathrm{LN}>30 \%$, liver $\leq 30 \%(\mathrm{n}=27)$; all $\leq 30 \%(\mathrm{n}=107)$. (C) Due to the high inter- 
lesion heterogeneity, the response patterns of the total tumor sizes could not fully reflect the individual lesion response in the mCRC patients. The response dynamics of the liver lesions were more associated with survival than those of other organs in the mCRC patients. 
Table 1.

Patient demographic information.

\begin{tabular}{|c|c|}
\hline Demographics & Total $(n=2,802)$ \\
\hline Male, n (\%) & $1,688(60.2)$ \\
\hline Age (years), mean (sd) & $60.74(10.91)$ \\
\hline White or Caucasians, n (\%) & $2,611(93.2)$ \\
\hline BSA, mean (sd) & $1.87(0.24)$ \\
\hline LDH baseline $\geq 1.5 \mathrm{UNL}, \mathrm{n}(\%)$ & $836(29.8)$ \\
\hline \multicolumn{2}{|l|}{ ECOG performance, $\mathrm{n}(\%)$} \\
\hline 0 & $1,536(54.8)$ \\
\hline 1 & $1,178(42.0)$ \\
\hline 2 & $87(3.2)$ \\
\hline \multicolumn{2}{|l|}{$K R A S$ status, $\mathrm{n}(\%)$} \\
\hline Wild-type & $795(28.4)$ \\
\hline Mutant & $593(21.2)$ \\
\hline Unknown & $1,414(50.4)$ \\
\hline \multicolumn{2}{|l|}{ Primary tumor type, n (\%) } \\
\hline Colon & $1910(68.2)$ \\
\hline Rectum & $892(31.8)$ \\
\hline \multicolumn{2}{|l|}{ Number of metastatic sites, $\mathrm{n}(\%)$} \\
\hline 1 & $382(13.6)$ \\
\hline 2 & $449(16.0)$ \\
\hline 23 & $1971(70.4)$ \\
\hline Liver-metastases only, n (\%) & $1347(48.1)$ \\
\hline Prior surgery, n (\%) & $2535(90.5)$ \\
\hline Prior radiation therapy, n (\%) & $367(13.1)$ \\
\hline Previous adjuvant therapy, n (\%) & $505(18.0)$ \\
\hline \multicolumn{2}{|l|}{ RECIST response, $\mathrm{n}(\%)$} \\
\hline Complete response & $77(2.7)$ \\
\hline Partial response & $1,069(38.2)$ \\
\hline Stable disease & $1,173(41.9)$ \\
\hline Progressive disease & $401(14.3)$ \\
\hline Not detected & $31(1.2)$ \\
\hline Unable evaluate & $39(1.4)$ \\
\hline Unknown & $12(0.3)$ \\
\hline \multicolumn{2}{|l|}{ Treatment, n (\%) } \\
\hline Bevacizumab plus chemotherapy & $377(13.5)$ \\
\hline Bevacizumab plus panitumumab plus chemotherapy & $371(13.2)$ \\
\hline FOLFIRI & $419(15.0)$ \\
\hline Panitumumab plus FOLFIRI & $425(15.2)$ \\
\hline FOLFOX & $769(27.4)$ \\
\hline Panitumumab plus FOLFOX & $441(15.7)$ \\
\hline
\end{tabular}

Cancer Res. Author manuscript; available in PMC 2021 November 01. 